\title{
IMPACT OF EXERCISE TRAINING ON HEMATOLOGICAL OUTCOMES FOLLOWING HEMATOPOIETIC CELL TRANSPLANTATION: A SCOPING REVIEW
}

Joseph Aziz, MD ${ }^{1 *}$, Chloe Smith, BSc ${ }^{2 *}$, Mitchell Slobodian, BSc ${ }^{4}$, Iris Du, BSc², Risa Shorr, BSc ${ }^{6}$, Michael De Lisio, PhD ${ }^{1,2,3,}$ David S. Allan, MD ${ }^{1,4,5}$

1 Departments of Medicine, 2 Cellular and Molecular Medicine, Faculty of Medicine, and 3 School of Human Kinetics, Faculty of Health Sciences, University of Ottawa, Ottawa, ON, Canada

4 Clinical Epidemiology Program, Ottawa Hospital Research Institute

5 Transplantation and Cell Therapy Program, Department of Medicine, and

6 Library Services, The Ottawa Hospital, Ottawa, ON, Canada

Both authors contributed equally

Submitted 2nd October 2020, Accepted 11th April 2021

Clin Invest Med 2021;44(2):E19-26; DOI: 10.25011/cim.v44i2.36369

\begin{abstract}
Purpose: Hematopoietic cell transplantation (HCT) is associated with significant risk prior to hematopoietic engraftment. Endurance exercise can modify the bone marrow microenvironment, alter hematopoiesis and accelerate hematopoietic regeneration in mouse models of transplantation.

Methods: A systematic review was conducted to clarify the impact of exercise on clinically relevant hematological outcomes in patients following HCT.

Results: A systematic search of the literature identified 13 studies (total of 615 participants; 313 in study arms). Studies included exercise regimens that were primarily low-to-moderate intensity. A total of five studies reported on engraftment and length of stay, which were largely unchanged with intervention. Rates of graft-versus host disease were reported in six studies whereas red cell and platelet transfusion needs were reported in four studies, neither of which was different with exercise. Survival was reported in four studies and was significantly improved by exercise in one study.

Conclusions: Exercise in patients receiving HCT appears feasible and safe. Heterogeneity in type and intensity of exercise was observed and few studies examined high intensity exercise. Outcome reporting was inconsistent regarding transplant-related outcomes. Standardized hematological outcome measures are needed to clarify the impact of higher intensity exercise on HCT.
\end{abstract}

Correspondence to: David Allan Email: daallan@toh.ca

\section{INTRODUCTION}

Hematopoietic cell transplantation (HCT) is used to treat patients with a variety of hematological malignancies and other blood, immune-related and metabolic disorders [1]. HCT can be performed using a patient's own hematopoietic cells for reconstitution (autologous HCT) or using cells from an immune-compatible healthy donor (allogeneic $\mathrm{HCT}$ ), depending on the underlying condition. Both types of transplants involve a period of profound cytopenia caused by the pre-transplant conditioning therapy that is associated with significant risk of infection and/or bleeding prior to initial hema- topoietic engraftment. The bone marrow microenvironment is often altered by the underlying disease [2-4], by treatments such as chemotherapy and radiation [5], and by associated co-morbidities and age, which can exacerbate delays in hematopoietic recovery and increase transplant-related complications [6]. Methods of restoring the health and supporting function of the bone marrow microenvironment-to accelerate engraftment and enhance competitive repopulation of specialized stem cell niches with blood-forming stem cells from the incoming graft-are needed [7]. Pre-clinical studies of treadmill exercise in mouse models demonstrated 
increased bone marrow cell survival and proliferation of hematopoietic stem cells suggesting increased quality of supporting bone marrow niches [8,9]. Additional studies have demonstrated a survival benefit and increased total blood reconstitution in mice that were pre-conditioned with exercise, although short and long-term donor engraftment was unchanged when compared with sedentary mice [10]. Clinical studies have demonstrated that exercise training interventions are safe and feasible in patients undergoing HCT [11]. Further, these studies have consistently shown improvements in quality of life, fatigue and physical performance [11]. However, the extent to which exercise can improve hematological outcomes for patients following HCT is rarely examined, and when examined it is often as a secondary outcome, and these results have never been succinctly summarized. Thus, a systematic scoping review of the literature is needed to understand the range of exercise intensity that has been reported in patients undergoing HCT and to understand the extent to which exercise can improve rates of hematopoietic engraftment and other clinically relevant transplant-related outcomes.

\section{METHODS}

This study's protocol was registered on PROSPERO (CRD42018106537, 29 August 2018). This report was prepared in accordance with the PRISMA guidelines for reporting scoping reviews [12].

\section{Eligibility criteria}

Eligibility criteria were developed $a$ priori to determine which full-text articles would be eligible for inclusion in this systematic review. Study population, intervention, comparator, outcome and study design (PICOS) parameters were predefined to allow for objective, reproducible analysis, as is standard for systematic reviews.

Population: Studies included for analysis enrolled patients undergoing autologous or allogeneic transplantation.

Intervention: Patients that enrolled in an exercise program as their primary intervention were included. Studies solely examining the use of exercise for non-hematological outcomes (e.g., quality of life, fitness, strength testing) or if the exercise was not performed during the immediate post-transplant period before engraftment were excluded. Studies that relied on self-reported exercise or physical activity participation were excluded.

Comparator: Any type of comparator was included. Non-comparative studies were excluded.

Outcome: Studies needed to include at least one hematological outcome, such as complete blood cell counts pre- and/or post-transplant, rates of engraftment, trans- fusion requirements, graft versus host disease (GVHD), and overall survival. Outcomes related to in vitro, ex vivo or invertebrate pre-clinical animal models were excluded.

\section{Study design}

All English language, controlled interventional studies (randomized, quasi-randomized and non-randomized) comparing exercise to placebo or a "no treatment" arm in clinical studies of patients undergoing HCT were included. Pre-clinical studies were excluded along with reviews, editorials, unpublished abstracts and records in a language other than English.

\section{Literature search strategy}

A comprehensive literature search strategy was developed in collaboration with a medical librarian (RS) specializing in systematic review searches and was peer reviewed by a second information specialist. The search strategy was used to search OVID MEDLINE, including In-Process \& Other Non-indexed Citations (Medline ALL), and OVID Embase Classic and Embase between January 1946 and June 22, 2018. The detailed search strategy is available from author upon request. The reference lists of included studies and any relevant reviews identified by the search strategy were reviewed to ensure that all relevant articles were captured.

\section{Study selection process}

All identified citations were imported into Covidence (Covidence Software, Melbourne, Australia) for management of search records, after removing duplicates. Articles were then screened in duplicate by independent reviewers (CS, ID, MS) based on title and abstract and the full texts of any potentially relevant studies were screened to determine final eligibility. In cases of disagreement between reviewers, consensus was achieved through discussion with a third team member (DA or MD). The study selection process was summarized using a flow diagram, as per PRISMA recommendations (Figure 1).

\section{Data extraction and analysis}

All included studies underwent data extraction in duplicate using a standardized electronic form in Covidence (www.covidence.org, Australia). The primary outcome of this study was to describe the effect of exercise training on engraftment in the context of HCT. Secondary outcomes included evaluating length of stay, rate of infection and GVHD, and overall survival. Descriptive statistics were used and $p$ values $<0.05$ were considered significant for direct comparisons. 


\section{FIGURE 1.}

PRISMA DIAGRAM OUTLINING THE IDENTIFICATION OF ARTICLES INCLUDED IN THE ANALYSIS.

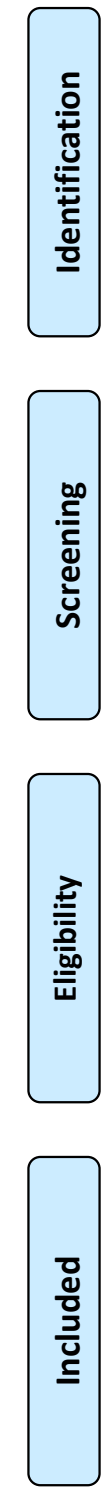

\section{RESULTS}

A total of 1,392 records were identified in our search performed on June 1, 2018 and two additional articles were identified from bibliographic review. After the removal of duplicates, 1,394 articles underwent screening of title and abstract to determine potential relevance. A total of 95 studies were retained and assessed for full-text eligibility. A further 82 studies were excluded for the following reasons: did not study patients undergoing transplant (54 studies); transplant outcomes were not included (21 studies); pre-clinical trial (four studies); exercise intervention not during transplant (two studies); and article not found (one study). A total of thirteen [13-25] studies were included in our analysis. A detailed summary of the study selection can be found in Figure 1.
The 13 studies that met the inclusion criteria described a total of 615 participants (54\% male) of which 313 were in the experimental group (see Table 1). Eleven [13-20,22,24,25] studies featured an adult population whereas two studies $[21,23]$ were performed in the pediatric setting. There were a similar number of studies using allogeneic (seven studies) $[14,15,18,19,21,23,25]$ and autologous (five studies) $[16,17,20,22,24]$ HCT, with one additional study [13] enrolling patients using autologous or allogeneic HCT. The primary source of cells was peripheral blood progenitor cells (five studies) [17,20$22,24]$, whereas four studies $[14,15,19,25]$ enrolled patients receiving either peripheral blood progenitor cells or bone marrow-derived cells, and four studies $[13,16,18,23]$ did not describe the cell source. Studies included pa- 
TABLE 1.

\section{CHARACTERISTICS OF PATIENTS ENROLLED IN CLINICAL STUDIES OF EXERCISE IN HEMATOPOIETIC CELL TRANSPLANTATION.}

\begin{tabular}{|c|c|}
\hline Transplant characteristics & Demographic data \\
\hline Number of studies included, $n$ & 13 \\
\hline Total participants, n (\% male) & $615(54)$ \\
\hline Intervention arm & 313 \\
\hline Control arm & 302 \\
\hline \multicolumn{2}{|l|}{ Population studied, (number of studies) } \\
\hline Adult & 11 \\
\hline Pediatric & 2 \\
\hline \multicolumn{2}{|c|}{ Diagnoses included in studies (number of studies) } \\
\hline Leukemia & 10 \\
\hline Lymphoma & 7 \\
\hline Aplastic anemia & 5 \\
\hline Multiple myeloma & 4 \\
\hline Breast carcinoma & 2 \\
\hline Other & 12 \\
\hline \multicolumn{2}{|l|}{ Type of donor used, (number of studies) } \\
\hline Allogeneic & 7 \\
\hline Autologous & 5 \\
\hline Mixed autologous and allogeneic & 1 \\
\hline \multicolumn{2}{|l|}{ Source of cells, (number of studies) } \\
\hline Peripheral blood progenitors & 5 \\
\hline Bone marrow & 0 \\
\hline Bone marrow OR peripheral blood & 4 \\
\hline Not described & 4 \\
\hline
\end{tabular}

tients with a diagnosis of leukemia (10 studies), lymphoma (seven studies), aplastic anemia (five studies), multiple myeloma (four studies), and most studies included patients across a broad range of diagnoses. With respect to intervention, most exercise programs featured low intensity ( $<10$ on Borg Scale [26]; five studies) $[15-17,19,20]$, seven studies used moderate intensity programs (10-13 on Borg Scale) [13,14,18,21,23-25], and only one study [22] used a high intensity program ( $>13$ on Borg Scale). Exercise modalities were variable but most commonly included stretching (six studies), resistance (six studies) and aerobic exercises (five studies). The intervention was performed after HCT in seven studies $[14,15,17,19,20,22,23]$, whereas six studies $[13,16,18,21,24,25]$ reported the intervention was performed both before and after transplantation. The majority of studies (12 studies) featured hospital-based, supervised interventions (see Table 2).
TABLE 2.

EXERCISE INTERVENTIONS AND TIMING IN CLINICAL STUDIES FEATURING EXERCISE PROGRAMS IN THE CONTEXT OF STEM CELL TRANSPLANTATION.

\begin{tabular}{|c|c|}
\hline Intervention & Studies, $\mathbf{n}$ \\
\hline \multicolumn{2}{|l|}{ Exercise modality } \\
\hline Stretching & 6 \\
\hline Resistance & 6 \\
\hline Aerobic & 5 \\
\hline Stationary bike & 4 \\
\hline Physiotherapy & 2 \\
\hline Breathing exercises & 2 \\
\hline Other & 8 \\
\hline \multicolumn{2}{|l|}{ Exercise intensity } \\
\hline Low & 5 \\
\hline Moderate & 7 \\
\hline Moderate to high & 1 \\
\hline \multicolumn{2}{|c|}{ Hospital based and supervised } \\
\hline Yes & 12 \\
\hline No & 1 \\
\hline \multicolumn{2}{|l|}{ Timing in relation to $\mathrm{HCT}$} \\
\hline Before & 0 \\
\hline After & 7 \\
\hline Before and after & 6 \\
\hline
\end{tabular}

Abbreviation: HCT, hematopoietic cell transplant

Among the 13 studies, outcomes were reported using a range of hematologic outcomes including days to engraftment, hemoglobin and platelet counts and transfusions, as well as length of stay, survival, infection and rates of graft versus host disease (GVHD). The outcomes reported in each study are summarized in Table 3. Five studies $[13,14,20,21,23]$ reported on engraftment, which was variably defined (first day that absolute neutrophil counts (ANC) $>0.5 \times 10^{9} / \mathrm{L}$ vs. first of three consecutive days with ANC $>0.5 \times 10^{9} / \mathrm{L}$ ) or not specifically defined (see Table 4). Baumann and colleagues [13] reported a significantly reduced time to neutrophil engraftment in the exercise group, whereas the four other studies $[14,20,21,23]$ reported no significant difference. A total of four studies $[20,21,23,24]$ reported similar red cell and platelet transfusion requirements in the exercise and usual treatment arms, although Dimeo and colleagues [20] noted a trend to reduced number of platelet transfusions in the exercise arm $(p=0.06)$. Another five studies $[13,14,17,20,21]$ evaluated length of hospital stay for patients (see Table 5). Dimeo and colleagues [20] demonstrated a statistically significant decrease 
TABLE 3.

\section{LIST OF REPORTED OUTCOMES IN CLINICAL STUDIES EVALUATING EXERCISE PROGRAMS IN THE CONTEXT OF HEMATOPOIETIC CELL TRANSPLANTATION.}

\begin{tabular}{|c|c|c|c|c|c|c|c|c|c|c|c|c|c|}
\hline $\begin{array}{l}\text { Study } \\
\text { (reference) }\end{array}$ & $\begin{array}{c}\text { Engraft- } \\
\text { ment } \\
\text { days }\end{array}$ & $\begin{array}{c}\text { Hb @ } \\
\text { admission }\end{array}$ & $\begin{array}{c}\text { Hb @ } \\
\text { discharge }\end{array}$ & $\begin{array}{c}\text { WBC @ } \\
\text { admission }\end{array}$ & $\begin{array}{c}\text { WBC @ } \\
\text { discharge }\end{array}$ & $\begin{array}{c}\text { PLT @ } \\
\text { admission }\end{array}$ & $\begin{array}{c}\text { PLT @ } \\
\text { discharge }\end{array}$ & $\begin{array}{l}\text { Red cell } \\
\text { transf. }\end{array}$ & PLT transf. & LOS & Survival & Infection & GVHD \\
\hline Baumann (13) & • & $\bullet$ & $\bullet$ & $\bullet$ & $\bullet$ & $\bullet$ & $\bullet$ & - & - & $\bullet$ & $\bullet$ & $\bullet$ & $\bullet$ \\
\hline Chamorro-Vina (21) & $\bullet$ & - & - & $\bullet$ & $\bullet$ & - & - & $\bullet$ & $\bullet$ & $\bullet$ & - & - & $\bullet$ \\
\hline Chamorro-Vina (23) & $\bullet$ & - & - & $\bullet$ & $\bullet$ & - & - & - & - & - & $\bullet$ & - & $\bullet$ \\
\hline Dimeo (17) & - & - & $\bullet$ & - & - & - & - & - & - & $\bullet$ & - & - & - \\
\hline Dimeo (20) & $\bullet$ & $\bullet$ & $\bullet$ & - & - & - & - & $\bullet$ & $\bullet$ & $\bullet$ & - & $\bullet$ & - \\
\hline Hayes (22) & - & - & - & $\bullet$ & $\bullet$ & - & - & - & - & - & - & - & - \\
\hline Jarden (14) & - & - & - & - & - & - & - & $\bullet$ & $\bullet$ & $\bullet$ & $\bullet$ & - & $\bullet$ \\
\hline Keser (16) & - & $\bullet$ & $\bullet$ & $\bullet$ & - & $\bullet$ & - & - & - & - & - & - & - \\
\hline Kim (15) & - & - & - & $\bullet$ & - & - & - & - & - & - & - & - & - \\
\hline Kim (19) & - & - & - & $\bullet$ & $\bullet$ & - & - & - & - & - & - & - & - \\
\hline Takekiyo (18) & - & $\bullet$ & $\bullet$ & - & - & - & - & - & - & - & - & - & $\bullet$ \\
\hline Coleman (24) & - & $\bullet$ & $\bullet$ & - & - & - & - & $\bullet$ & $\bullet$ & - & - & - & - \\
\hline Wiskemann (25) & - & - & - & - & - & - & - & - & - & - & $\bullet$ & - & $\bullet$ \\
\hline Total & 5 & 5 & 6 & 7 & 7 & 3 & 2 & 4 & 4 & 5 & 4 & 4 & 6 \\
\hline
\end{tabular}

Abbreviations: GVHD, graft versus host disease; LOS, length of stay (in hospital); PLT, platelet transfusion; WBC, white blood cell

TABLE 4

\section{ENGRAFTMENT AND TRANSFUSION OUTCOMES IN CLINICAL STUDIES OF EXERCISE IN THE CONTEXT OF HEMATOPOIETIC CELL TRANSPLANTATION.}

\begin{tabular}{|c|c|c|c|c|c|c|c|}
\hline Study (reference) & Exercise intensity* & Engraft days (d) & $p$ & RBC transf (units) & $p$ & PLT Transfusion (units) & $p$ \\
\hline Dimeo (20) & $\mathrm{L}$ & $6.6 / 7.6^{+}$ & 0.01 & $3.3 / 3.3^{\ddagger}$ & 0.9 & $19.5 / 26.9^{\ddagger}$ & 0.06 \\
\hline Chamorro-Vina (21) & M & $13 / 13$ & $>0.05$ & $2.0 / 1.6$ & $>0.05$ & $2.7 / 2.1$ & $>0.05$ \\
\hline Jarden (14) & M & $22.1 / 20.4^{5}$ & 0.3 & $7 / 5.5$ & 0.3 & $13 / 8$ & 0.16 \\
\hline Baumann (13) & M & 15.5 / 15.9 & 0.8 & - & - & - & - \\
\hline Chamorro-Vina (23) & M & $12 / \mathrm{NR}^{\S}$ & $>0.05$ & - & - & - & - \\
\hline Coleman (24) & $M$ & - & - & $\begin{array}{l}1.8 / 2.3 \\
1.0 / 1.8\end{array}$ & $F=2.57$ & $\begin{array}{l}2.3 / 3.1 \\
2.0 / 3.6\end{array}$ & $F=4.11$ \\
\hline
\end{tabular}

Abbreviations: ANC, absolute neutrophil counts; F, F statistic; NR, not reported; PLT, platelet transfusions; RBC, red blood cell

Median for intervention/control groups are presented.

"Exercise intensity where $L$ represents low intensity, $M$ represents moderate intensity

${ }^{\dagger}$ Engraftment defined as $1^{\text {st }}$ day of ANC $>0.5 R B C$ and PLT defined as number of units transfused

${ }^{\ddagger} R B C$ and PLT defined as number of units transfused

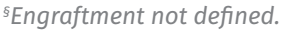

"Engraftment defined as 3 days consecutive of ANC $>0.5$

$p>0.05$ reported in studies as non-significant, no raw $p$ value provided

in length of hospitalization ( $13.6 \pm 2.2$ days vs. $15.2 \pm 3.6$ days) whereas the other studies $[13,14,17,21]$ reported no significant differences. A study by Wiskemann and colleagues [25] reported improved survival in patients who were enrolled in an exercise program (see Table 5) with $66 \%$ survival at two years vs. $49.1 \%$ in the usual care $\mathrm{arm}$. This study featured a male-predominant population who underwent a moderate intensity exercise intervention and enrolled patients with leukemia (acute myeloid or lymphoid leukemia and treatment-related acute leukemia). Three other studies [13,14,23] evaluated overall survival and did not report any significant differences between the study arms. The studies by Jarden and colleagues [14] and Baumann and colleagues [13] evaluated similar populations to that of Wiskemann and colleagues [25], whereas the study by Chamorro-Viña 
TABLE 5.

\section{SECONDARY OUTCOMES IN CLINICAL STUDIES OF EXERCISE PROGRAMS IN THE CONTEXT OF HEMATOPOIETIC} CELL TRANSPLANTATION*.

\begin{tabular}{|c|c|c|c|c|c|c|c|c|c|}
\hline Study (reference) & Exercise intensity $^{\dagger}$ & LOS (days) & p & Survival (\%) & $p$ & Infection (\%) & p & GVHD (\%) & $p$ \\
\hline Dimeo (17) & $\mathrm{L}$ & $20 / 18$ & $>0.05$ & & & & & & \\
\hline Dimeo (20) & $\mathrm{L}$ & $13.6 / 15.2$ & 0.03 & & & $18.2 / 16.2$ & $\mathrm{n} / \mathrm{a}$ & & \\
\hline Chamorro-Vina (21) & M & $16 / 16$ & $>0.05$ & & & & & $29 / 46$ & $\mathrm{nr}$ \\
\hline Jarden (14) & M & $34.7 / 35$ & 0.884 & $90.5 / 95.2$ & $\mathrm{nr}$ & & & $23.8 / 42.9$ & 0.326 \\
\hline Baumann (13) & M & $41 / 43$ & $\mathrm{n} / \mathrm{a}$ & $75 / 78.1$ & $\mathrm{nr}$ & $15.6 / 9.4$ & $\mathrm{n} / \mathrm{a}$ & $65.6 / 50$ & $\mathrm{nr}$ \\
\hline Chamorro-Vina (23) & M & & & $100 / 33.3$ & $\mathrm{nr}$ & & & $66.7 / 33.3$ & $\mathrm{nr}$ \\
\hline Takekiyo (18) & M & & & & & $41.2 / 44.4$ & $\mathrm{n} / \mathrm{a}$ & $-/ 50$ & $\mathrm{nr}$ \\
\hline Wiskemann (25) & $M$ & & & $66.0 / 49.1$ & 0.112 & $10.0 / 18.9$ & $\mathrm{n} / \mathrm{a}$ & $44.0 / 37.7$ & 0.55 \\
\hline
\end{tabular}

Abbreviations: GVHD, graft versus host disease; LOS, length of stay (in hospital); $\mathrm{nr}$ represents p value not reported in manuscript

Median for intervention/control groups are presented.

${ }^{\dagger}$ Exercise intensity where L, M and $\mathrm{H}$ represent low, moderate and high intensity, respectively.

and colleagues [23] was performed in the pediatric setting and included only six participants. Infection rates were evaluated in four studies $[13,18,20,25]$ and no significant differences were observed between the exercise and control arms (Table 5). Lastly, six studies $[13,14,18,21,23,25]$ evaluated the overall incidence of GVHD in the post-transplant period (Table 5). Although several studies $[14,18,21]$ reported a lower incidence of GVHD in the exercise arms, none of these were statistically significant.

\section{DISCUSSION}

Our scoping review highlights that patients undergoing HCT can participate in a range of exercise programs of low to high intensity, which appear feasible and safe across the spectrum of underlying haematological malignancies and related blood disorders. Based on the available literature, the impact of low to moderate exercise on outcomes following transplantation, however, appears negligible. While one study [25], using a moderate intensity exercise program, revealed an overall survival benefit, there is currently no clear evidence that exercise can accelerate engraftment or reduce transfusion requirements in patients undergoing autologous or allogeneic HCT. Several caveats should be considered when evaluating these studies, which include the generally low sample size and heterogeneity of exercise protocols. In addition, standardized outcome reporting was generally lacking and would have allowed for pooling of data for more meaningful and insightful meta-analysis regarding the impact of exercise on survival, engraftment, transfusion needs and relapse. Future studies should address exercise programs of greater intensity, as most studies utilized stretching and breathing exercises that were not reflective of the treadmill running used in preclinical models, which revealed changes in bone marrow composition [10].
Despite the overall lack of clear benefit, favourable outcomes were reported by several studies, which suggest additional investigation regarding the benefits of exercise on hematological outcomes following HCT is warranted. Of note, the PETRA study, a randomized controlled trial which completed enrolment in June 2019 (www.clinicaltrials.gov; NCT01374399), evaluated the effect of exercise on overall survival in allogeneic HCT but remains unpublished. Although physical activity in HCT survivors has demonstrated physical and psychological benefits [27], exercise guidelines specifically designed to improve clinically relevant transplant-specific outcomes are lacking due to insufficient evidence. As per the American College of Sports Medicine (ACSM), moderate to vigorous intensity exercise is recommended for improving performance and psychological outcomes in cancer survivors with insufficient evidence from clinical trials to produce guidelines for improving clinical outcomes concerning the underlying cancer or treatment [28]. Importantly, current ACSM guidelines are based primarily on patients with early-stage breast, prostate and colorectal cancer. Thus, the intensity recommendations may not be directly applicable to patients with hematological cancer undergoing HCT as these patients have unique alterations in their underlying physiology related to oxygen-carrying capacity and treatment-related side effects [29]. The growing body of literature investigating exercise programs in HCT should address this knowledge gap.

The limited research on exercise in patients undergoing $\mathrm{HCT}$, which was summarized in two recent meta-analyses, indicates that exercise improves patients' quality of life [30,31]. This includes increased cardiorespiratory fitness, decreased fatigue and improved physical, emotional and cognitive functioning. Keser and colleagues [32] also suggested possible improved mobilization of stem cells with exercise therapy prior to autologous 
stem cell transplant providing a distinct avenue for investigation that may benefit patients undergoing HCT by improving the quality of the graft [33]. Despite the benefits of exercise in some patient groups and improved hematological outcomes in pre-clinical studies [10], mechanistic insight regarding the impact of exercise on engraftment and potential improvements in survival remains lacking.

\section{Limitations}

Our study has some limitations that should be acknowledged. As with any systematic review, the possibility of relevant articles not being identified within our search strategy remains. Moreover, the heterogeneity of outcome reporting and exercise interventions has limited our ability to pool data and make meaningful conclusions with respect to the efficacy of the intervention. More standardized study designs and outcome reporting is needed in future studies to further assess the efficacy of exercise programs in the context of HCT.

\section{CONCLUSIONS}

Overall, exercise training in the setting of autologous and allogeneic stem cell transplantation appears to be safe and feasible in the setting of a variety of hematologic disorders. The impact of exercise on engraftment, although promising in some murine models, remains unclear in the clinical setting. The heterogeneity of study design and exercise intensity limits our ability to comment definitively on the overall benefits of exercise in the context of engraftment and transplant-related outcomes such as overall survival, GVHD and infection rates. Exercise regimens of higher intensity should be investigated to assess the impact of exercise in the setting of HCT.

\section{FINANCIAL SUPPORT}

This work was supported by funds from the Natural Sciences and Engineering Research Council of Canada and the American Institute for Cancer Research (MD).

\section{ACKNOWLEDGEMENTS}

The authors have no conflicts of interest to disclose.

\section{REFERENCES}

1. Majhail NS, Farnia SH, Carpenter PA, et. al. Indications for autologous and allogeneic hematopoietic cell transplantation: guidelines from the American Society for Blood and Marrow Transplantation. Biol Blood Marrow Transplant. 2015; 21: 1863-1869.

2. Chandran P, Le Y, Li Y, et al. Mesenchymal stromal cells from patients with acute myeloid leukemia have altered capacity to expand differentiated hematopoietic progenitors. Leuk Res. 2015; 39: 486-493.
3. Kumar B, Garcia M, Weng L, et al. Acute myeloid leukemia transforms the bone marrow niche into a leukemia-permissive microenvironment through exosome secretion. Leukemia. 2018; 32: 575-587.

4. Roccaro AM, Sacco A, Maiso P, et al. BM mesenchymal stromal cell-derived exosomes facilitate multiple myeloma progression. J Clin Invest. 2013; 123: 1542-1555.

5. Duarte D, Hawkins ED, Akinduro O, et al. Inhibition of endosteal vascular niche remodeling rescues hematopoietic stem cell loss in AML. Cell Stem Cell. 2018; 22: 64-77.

6. Emmons R, Niemiro GM, De Lisio M. Hematopoiesis with obesity and exercise: role of the bone marrow niche. Exerc Immunol Rev. 2017; 23: 82-95.

7. Allan DS, De Lisio M. Reversing pathological remodelling of the bone marrow microenvironment in acute myeloid leukemia. Stem Cell Invest. 2018; 5: 29. doi: 10.21037/ sci.2018.09.02.

8. Emmons R, Ngu M, Xu G, Hernández-Saavedra D, Chen H, de Lisio M. Effects of Obesity and Exercise on Bone Marrow Progenitor Cells after Radiation. Med Sci Sports Exerc. 2019; 51: 1126-1136.

9. Emmons R, Niemiro GM, Owolabi O, De Lisio M. Acute exercise mobilizes hematopoietic stem and progenitor cells and alters the mesenchymal stromal cell secretome. J Appl Physiol. 2016; 120: 624-632.

10. De Lisio M, Baker JM, Parise G. Exercise promotes bone marrow cell survival and recipient reconstitution postbone marrow transplantation, which is associated with increased survival. Exp Hematol. 2013; 41:143-154.

11. Wiskemann J, Huber G. Physical exercise as adjuvant therapy for patients undergoing hematopoietic stem cell transplantation. Bone Marrow Transplant. 2008; 41: 321-329.

12. Tricco AC, Lillie E, Zarin $W$ et al. PRISMA extension for scoping reviews (PRISMA-SCR): Checklist and explanation. Ann Intern Med. 2018; 169: 467-473.

13. Baumann FT, Kraut L, Schüle K, Bloch W, Fauser AA. A controlled randomized study examining the effects of exercise therapy on patients undergoing haematopoietic stem cell transplantation. Bone Marrow Transplant. 2010; 45: 355-362.

14. Jarden M, Baadsgaard MT, Hovgaard DJ, Boesen E, Adamsen L. A randomized trial on the effect of a multimodal intervention on physical capacity, functional performance and quality of life in adult patients undergoing allogeneic SCT. Bone Marrow Transplant. 2009; 43:725-737.

15. Kim SD, Kim HS. A series of bed exercises to improve lymphocyte count in allogeneic bone marrow transplantation patients. Eur J Cancer Care. 2006; 15: 453-457.

16. Keser I, Suyani E, Yosmaoglu HB, Aki SZ, Turkoz Sucak AG. Acute physiological responses to physiotherapy applications pre and post autologous stem cell transplantation: an experimental study. Hematology. 2014; 19: 136-140.

17. Dimeo FC, Tilmann MH, Bertz H, Kanz L, Mertelsmann R, Keul J. Aerobic exercise in the rehabilitation of cancer patients after high dose chemotherapy and autologous peripheral stem cell transplantation. Cancer. 1997; 79: 17171722. 
18. Takekiyo T, Dozono K, Mitsuishi T, et al. Effect of exercise therapy on muscle mass and physical functioning in patients undergoing allogeneic hematopoietic stem cell transplantation. Supportive Care Cancer. 2015; 23: 985-992.

19. Kim SD, Kim HS. Effects of a relaxation breathing exercise on anxiety, depression, and leukocyte in hemopoietic stem cell transplantation patients. Cancer Nursing. 2005; 28: 79-83.

20. Dimeo F, Fetscher S, Lange W, Mertelsmann R, Keul J. Effects of aerobic exercise on the physical performance and incidence of treatment-related complications after highdose chemotherapy. Blood. 1997; 90: 3390-3394.

21. Chamorro-Vina C, Ruiz JR, Santana-Sosa E, et al. Exercise during hematopoietic stem cell transplant hospitalization in children. Med Sci Sports Exerc. 2010; 42: 1045-1053.

22. Hayes S, Rowbottom D, Davies P, Parker T, Bashford J. Immunological changes after cancer treatment and participation in an exercise program. Med Sci Sports Exerc. 2003; 35: 2-9.

23. Chamorro-Viña C, Valentín J, Fernández L, et al. Influence of a moderate-intensity exercise program on early NK cell immune recovery in pediatric patients after reduced-intensity hematopoietic stem cell transplantation. Integr Cancer Ther. 2017; 16: 464-472.

24. Coleman EA, Coon SK, Kennedy RL, et al. Effects of exercise in combination with epoetin alfa during high-dose chemotherapy and autologous peripheral blood stem cell transplantation for multiple myeloma. Oncol Nurs Forum. 2008; 35: E53-61. doi: 10.1188/08.ONF.E53-E61.

25. Wiskemann J, Kleindienst N, Kuehl R, Dreger P, Schwerdtfeger R, Bohus M. Effects of physical exercise on survival after allogeneic stem cell transplantation. Int J Cancer. 2015; 137: 2749-2756.
26. Borg GA. Psychophysical bases of perceived exertion. Med Sci Sports Exerc. 1982; 14: 377-381.

27. Wiskemann J, Huber G. Physical exercise as adjuvant therapy for patients undergoing hematopoietic stem cell transplantation. Bone Marrow Transplant. 2008; 41: 321-329.

28. Campbell KL, Winters-Stone KM, Wiskemann J, et al. Exercise guidelines for cancer survivors: consensus statement from international multidisciplinary roundtable. Med Sci Sports Exerc. 2019; 51: 2375-2390.

29. Kuehl R, Scharhag-Rosenberger F, Schommer K, et al. Exercise intensity classification in cancer patients undergoing allogeneic HCT. Med Sci Sports Exerc. 2015; 47: 889-895.

30. Van Haren IE, Timmerman H, Potting CM, Blijlevens NM, Staal JB, Nijhuis-van der Sanden MW. Physical exercise for patients undergoing hematopoietic stem cell transplantation: systematic review and meta-analyses of randomized controlled trials. Phys Ther. 2013; 93: 514-528.

31. Persoon S, Kersten MJ, van der Weiden K, et al. Effects of exercise in patients treated with stem cell transplantation for a hematologic malignancy: a systematic review and meta-analysis. Cancer Treat Rev. 2013; 39: 682-690.

32. Keser I, Suyani E, Aki SZ, Sucak AG. The positive impact of regular exercise program on stem cell mobilization prior to autologous stem cell transplantation. Transf Apher Sci. 2013; 49: 302-306.

33. Emmons R, Niemiro GM, De Lisio M. Exercise as an adjuvant therapy for hematopoietic stem cell mobilization. Stem Cells Int. 2016; 2016:7131359. doi: 10.1155/2016/7131359. 